\title{
Los nuevos imaginarios culturales en la ciudad de Tarragona: Un análisis de la distribución de sus locales
}

\author{
Jose Reche Navarro \\ Licenciado en Antropología, Universitat Rovira i Virgili \\ jose5reche@hotmail.com
}

Resumen: Aunque hay mucho escrito sobre el mundo de la llamada new age o "nebulosa místico-esotérica", no existe nada en lo referente a su distribución espacial en una ciudad. Este artículo trata de hacer una descripción y análisis de estas actividades, así como de los locales en los que tienen lugar, en el contexto de la ciudad de Tarragona. Para ello, se establece en primer lugar un análisis de los locales sobre la base de su tipología y de su ámbito temático (terapias naturales, espiritualidad oriental, esoterismo y neochamanismo). Luego se analiza su distribución en la ciudad y se propone una posible dinámica de implantación y crecimiento. Para ilustrarlo se ba recurrido al ejemplo de las espiritualidades orientales. Por último, se intenta extraer alguna conclusión de todo ello.

Palabras clave: Nuevos imaginarios culturales, terapias naturales, espiritualidades orientales, esoterismo, distribución urbana de centros relacionados con la espiritualidad new age.

Abstract: Though much has been written on the world of the new age or "mysticalesoteric nebula", nothing has been written on the way it spreads in a city. This article aims to describe and analyze these activities and the places in which they occur in Tarragona. In order to do this, first the different activities are analyzed in terms of their typology and thematic scope (natural therapies, Eastern spirituality, esotericism and neo-shamanism). The article then analyses their distribution throughout the city and proposes an implementation and growth dynamic. To illustrate this, the example of Eastern spirituality has been used. Finally, the study offers various conclusions that can be drawn from its findings.

Keywords: New cultural imaginaries, natural therapies, Eastern spirituality, esotericism, urban distribution of centres related to new age spirituality. 


\section{Introducción}

Aunque parezca algo reciente, el fenómeno al que se le han dado nombres tan diversos como new age (Heelas, 1996; Hanegraaff, 1998), nebulosa místicoesotérica (Champion, 1989; Prat y Moreno, 2007), religiosidad alternativa (Lenoir, 2003) o nuevos imaginarios culturales (GRIC, 2012) en realidad posee ya más de un siglo de existencia. Por una parte, sus raíces se hunden en el misticismo naturalista de los trascendentalistas americanos (R. W. Emerson, H. D. Thoreau y W. Whitman) y, por otra, en la teosofía de Helena P. Blavatsky, quien plasmó su ideología en dos voluminosas obras (1877 y 1888). Sin embargo, será esta última la que establecerá una serie de características que luego se repetirán, esta vez entre la gran masa, a partir de los años sesenta del siglo $\mathrm{xx}$.

Medio siglo antes de que Aldous Huxley (1945) formulase su filosofía perenne, la teosofía planteó ya la idea de una espiritualidad universal, pilar de la futura new age. Y no solo eso, sino que manejaba con comodidad y sin tapujos conceptos e imaginarios provenientes del esoterismo occidental (cábala, masonería, alquimia, magia, neoplatonismo, gnosticismo y druidismo) y de las religiones orientales (hinduismo, budismo, zoroastrismo y confucionismo). Popularizó conceptos como transmutación, karma, reencarnación, chakras, maya, mantra, kali yuga, tetragrammaton o sefirot y prácticas como la meditación o el mediumnismo (que evolucionaría hacia el actual channeling) que darían gran importancia a la mujer. Sin olvidar que fue el primer movimiento de este tipo que planteó acercar la ciencia y la religión mediante un modelo espiritual, intentando crear así una ciencia espiritual. Es decir, algo que trascendiese tanto a la ciencia materialista del xix, basada en un racionalismo estrecho, como a la tradicional religión cristiana, basada exclusivamente en la creencia. En resumen, estableció las estructuras básicas de la líquida espiritualidad laica que el Grup de Recerca sobre Imaginaris Culturals (GRIC), del que formo parte, ha denominado nuevos imaginarios culturales.

En la primera mitad del siglo xx la teosofía se dividió en corrientes como la Antroposofía de Rudolf Steiner (1978) o la Escuela Arcana de Alice Ann Bailey (1985), por citar solo un par de ellas. Estas continuaron ampliando su base social, pero sin llegar nunca a ser un fenómeno masivo. Para eso habría que esperar a la guerra fría. 
En la década de los sesenta del pasado siglo confluyeron una serie de factores, especialmente en EE. UU., que dieron lugar a una explosión social donde el modelo teosófico se vio ampliado y enriquecido, aunque no alterado en su estructura básica. Factores como la merma de derechos civiles por la guerra fría, la discriminación de minorías étnicas, el asesinato de J.F.K. o la guerra de Vietnam dieron lugar a que la autoridad de los gobiernos y sus ideologías afines, entre las que se hallaba el dogma cristiano, empezasen a perder el apoyo popular. Este aflojamiento de las estructuras ideológicas y religiosas tradicionales se vio reforzado por el surgimiento de la generación beat, las organizaciones marxistas clandestinas, el movimiento por los derechos civiles americano con el uso de la no violencia gandhiana o el mayo del 68 francés, que eclosionarían en el movimiento hippy y pacifista. Esta vez sí, como fenómeno de masas.

Es en ese terreno social de aflojamiento de la autoridad y las ideologías oficiales cuando el tipo de espiritualidad universalista, pero individualizada, de corte teosófico-esotérico extendió sus raíces a los amplios estratos medios de la sociedad, produciendo no solo el flower power, sino un verdadero florecimiento de nuevos ideales e imaginarios. Las diferencias básicas con el movimiento teosófico anterior fueron la irrupción con fuerza de las espiritualidades orientales y la incorporación de una corriente psicológica espiritual.

Si bien los contenidos orientales ya estaban presentes en la teosofía - pasados por el tamiz cognitivo de Blavatsky-, la llegada de maestros, lamas y yoguis que trasmitían sus enseñanzas directamente supuso una diferencia significativa. En efecto, la aprobación de la Ley de Inmigración Asiática en 1965 permitió la llegada de orientales a EE. UU., a los que se les había denegado la entrada desde 1924. A partir de entonces, las ideas, y sobre todo las prácticas indias, chinas y japonesas, fertilizarían ampliamente ese núcleo teosófico-esotérico original.

La otra gran diferencia con la primera mitad del siglo xx es la incorporación de terapias psicológicas. Su precedente se halla en el Movimiento del Potencial Humano impulsado por Abraham Maslow, Aldous Huxley y Alan Watts, y cuyo lugar más emblemático sería el Instituto Esalen, fundado por Michael Murphy y Richard Price, ambos psicólogos (Schwartz, 2002: 87-131). Allí se experimentaría con nuevas terapias para el desarrollo humano: psicología humanista, gestalt, rolfing, bioenergética, terapia primal, análisis transaccional, etc. Este movimiento psicológico daría lugar al surgimiento de una psicolo- 
gía abiertamente espiritual: la psicología transpersonal (Charles Tart, 1979; Stanislav Grof, 1994 y 2001; Ken Wilber, 1990 y 1993; Michael Washburn, 1999). Esta integra prácticas y conceptos tanto orientales como occidentales. Tampoco habría que olvidar la inclusión del psicoanálisis jungiano, pues gran parte de la terapéutica de los nuevos imaginarios culturales está impregnada de sus conceptos: constelaciones, sombra, arquetipos, shelf, ánima/animus, etc.

$\mathrm{Al}$ igual que la teosofía, también los nuevos imaginarios culturales han integrado conceptos y visiones provenientes de la ciencia, especialmente de la física (David Bohm, 2008; Fritjof Capra, 2005; Michio Kaku, 2010), la biología (James Lovelock, 2007 y 2008; Rupert Sheldrake, 1989) o la neurología (Karl Pribram, 1995), y, por supuesto, de la antropología (Carlos Castaneda, 1968; Michael Harner, 1980; Jeremy Narby, 1999). Aunque esta penetración de las ciencias ha necesitado siempre de unos buenos divulgadores, y casi cada década tiene su best seller para difundirlas. Si a principios del siglo xx podríamos señalar a El libro de los condenados de Charles Fort (1919), en los sesenta será El retorno de los brujos de Louis Pauwels y Jacques Bergier (1960), en los ochenta La conspiración de acuario de Marilyn Ferguson (1980), en los noventa El paradigma holográfico de Michael Talbot (1991) y en la primera década del siglo xxi ¿iY tú qué sabes!? de William Arntz, Betsy Chasse y Mark Vicente (2006) y Física de lo imposible de Michio Kaku (2010).

A partir de los sesenta se produjo también la irrupción del neochamanismo en un clima internacional de descolonización que se abría a la posibilidad del conocimiento proveniente de los pueblos nativos y en un ambiente social que desde la generación beat miraba el alcohol y las drogas con otros ojos. $\mathrm{Su}$ introductor y gran constructor teórico fue el antropólogo Carlos Castaneda, que en su tetralogía $(1968,1971,1972,1974)$ ejemplificó perfectamente las dos grandes corrientes metodológicas de este ámbito. Sus dos primeros libros, Las Enseñanzas de Don Juan (1968) y Una realidad aparte (1971) exploran y ejemplifican la corriente enteogénica, dedicada especialmente al autoconocimiento sobre la base del uso de psicotrópicos. Le seguirán en esta línea Terence McKenna $(1994,2001)$ y los actuales Christian Rätsch (2002), Daniel Pinchbeck $(2006,2007)$ y, más cerca de nosotros, Josep Ma Fericgla (1994, 1997). En Viaje a Ixtlán (1972) y Relatos de Poder (1974), Castaneda abandona esa vía y explora caminos alternativos más cercanos a las vías yóguicas. En esa línea alternativa seguirá Michel Harner (1980), también antropólogo, que en 
los años ochenta empezó a enseñar un neochamanismo de corte terapéutico al que le han seguido en nuestro país toda una cohorte de neochamanes latinoamericanos.

\section{Características generales de los nuevos imaginarios culturales y de sus practicantes}

Probablemente sea la amplitud, complejidad y liquidez del fenómeno lo que hizo que no fuese estudiado por las ciencias humanas hasta finales de los años ochenta, sobre todo a partir de los noventa. Esos primeros estudios vinieron de expertos en religión comparada (Lewis, 1992; Melton, 1992; Heelas, 1996), historiadores (Hanegraaff, 1998) y sociólogos (Champion, 1989, 1994; Barker, 1994; Lenoir, 2003). Los antropólogos se han sumado tarde al estudio de este fenómeno (Carozzi, 1999; Siqueira, 2004; Prat y Moreno, 2007; Fedele, 2008), si bien, y hasta donde conozco, es en este ámbito donde se ha dado el único trabajo de campo de conjunto (GRIC, 2012).

Tanto en los estudios sociológicos y antropológicos como en los de religión comparada se enumeran una serie de características generales de los nuevos imaginarios culturales que pueden variar ligeramente de un autor a otro: carácter mundano específico, holismo, evolucionismo, psicologización de la religión y sacralización de la psicología y esperanza en la llegada de una nueva era (Hanegraaff, 1998); fronteras muy tenues entre ámbitos y libre circulación de sus practicantes de uno a otro, énfasis en la experiencia, búsqueda de la felicidad en este mundo, visión monista de la realidad, optimismo en la capacidad automotora humana, ética del amor; rechazo de postulados abrahámicos como el dualismo o el sentido de culpa, importancia de los líderes carismáticos (Champion, 1993); paradigma holográfico, influencia de la psicología profunda y de la antropología (Lenoir, 2003). En cuanto al tipo de adepto de los nuevos imaginarios culturales, tanto para F. Champion (1989) como para D. Siqueira (2004), sería mayoritariamente femenino, de entre 30 y 50 años, de clase media, con estudios universitarios y trabajo sobre todo en los sectores de la enseñanza y el mundo sanitario, perfil que ha sido confirmado y ampliado por el GRIC (2012: 315-17). 


\section{Focos de atracción e irradiación}

Las grandes ciudades suelen ser los centros focales a partir de los cuales los nuevos imaginarios culturales irradian hacia ciudades más pequeñas y desde allí al ámbito rural, existiendo así toda una red de conexiones. En este fenómeno, como en otros, las ciudades de una región suelen estar conectadas con las de otras regiones, así como con otros países dentro del propio continente y fuera de este, habiéndose tejido toda una red planetaria que se ha ido espesando a medida que avanzaba la globalización. En esta red puede observarse la existencia de países, y especialmente ciertas áreas dentro de esos países, que se han convertido en focos de atracción e irradiación de técnicas, ideas y cosmovisiones. Los Estados Unidos destacan sobre los demás países y, dentro de este, los estados de California y Colorado. En Europa destacan el suroeste de Inglaterra (Glastonbury, Somerset y Cornualles) y Francia. Y en España sobresalen Cataluña y el País Vasco, en especial sus grandes ciudades. Asimismo, destacan otros lugares como el Pirineo oscense o las Alpujarras granadinas.

En el estudio realizado por el GRIC (2012) se hallaron conexiones diversas entre Tarragona y Barcelona. Hacia esta última ciudad se desplazan numerosos usuarios para realizar cursos y recibir servicios o formación en diversas técnicas. De esa misma ciudad llegan a Tarragona algunos profesionales para impartir sus servicios.

Se detectaron igualmente conexiones con otros lugares de Cataluña, especialmente de sus proximidades (Reus, Valls, Altafulla, Torredembarra, montañas de Prades, etc.), y también con puntos más alejados como Lleida o Santa Maria de Palautordera, al igual que con otras comunidades como Aragón (Panillo, Moncayo). Tarragona, según ese mismo estudio, tiene conexiones con países como Portugal (yoga), Italia (terapias naturales), Francia (budismo, yoga, terapias naturales), el Reino Unido (budismo), Canadá (yoga), los Estados Unidos (yoga), México (neochamanismo), Cuba (terapias naturales), Brasil (necochamanismo), Perú (neochamanismo) y la India (yoga).

\section{Objetivos y metodología}

La liquidez y nebulosidad de los nuevos imaginarios culturales conlleva que desde la antropología o la sociología se produzcan, bien estudios generales en- 
focados sobre todo a su visión del mundo, como los citados arriba, bien estudios de caso concretos (Ravinovitch y Reid, 2004; Fedele, 2008). El GRIC propuso un acercamiento diferente en Els nous imaginaris culturals (2012), un estudio de campo de conjunto en el marco del Camp de Tarragona. Se trataba con ello no solo de comprobar hasta qué punto se cumplían o no los planteamientos teóricos generales expuestos anteriormente, sino también de qué forma se concretaba eso en un marco físico concreto. Es en esta misma línea que se realizó una primera cartografía de los locales dedicados a actividades relacionadas con los nuevos imaginarios culturales en la ciudad de Tarragona, la cual, sin embargo, dado el volumen de material recogido, tuvo que ser eliminada de aquella obra. Fue así como se planteó la posibilidad de publicarla en forma de artículo.

Primeramente se confeccionó una tabla de todos los locales relacionados con el ámbito de los nuevos imaginarios culturales (centros, consultas, gimnasios, comercios, etc.), que hubo de ser actualizada dado el tiempo transcurrido entre la finalización de la investigación y el presente artículo, y donde aparecían el nombre del local y las actividades que allí se llevaban a cabo así como - siempre que fue posible - información de tipo práctico (teléfono, e-mail, página web, persona responsable, etc.). Dicha tabla no incluye ninguno de los barrios periféricos de la ciudad. Luego se procedió al recuento de los locales y a situarlos en el mapa, y a partir de aquí a la búsqueda de algún patrón en su distribución y a las posibles causas de ello. Por último, se ha introducido la variable histórica como factor comprobante de la dinámica de distribución propuesta, usando para ello el ámbito de las espiritualidades orientales por ser el que mejor domino. Es cierto que esa misma variable histórica sería más completa y explicativa si se aplicase también a los demás ámbitos estudiados (terapias alternativas, esoterismo y neochamanismo), pero desbordaría ampliamente el objetivo y la extensión de un artículo como este. Lo mismo sucede con un estudio detallado de sus conexiones con otras ciudades y países.

A efectos de clasificación de los diferentes tipos de actividades englobadas en los nuevos imaginarios culturales, he partido de la propuesta por el GRIC a partir de Vicente Merlo (2007), es decir, terapias, espiritualidad oriental y esoterismo, y he añadido el neochamanismo como cuarto ámbito. Tras las investigaciones realizadas hemos visto que, si bien el neochamanismo comparte con el esoterismo un tipo de visión mágica de la realidad, su fuente de conocimientos 
teóricos y prácticos no se halla en la remota antigüedad occidental, sino en un amplio abanico de culturas no occidentales donde el chamanismo ha constituido una esfera altamente influyente, cuando no su principal ámbito espiritual. Esto conlleva que, mientras que en el campo del esoterismo los usuarios se hallan más conectados e interesados por la magia, el ocultismo y la historia, los intereses de los neochamanes se desplacen hacia la sanación, el ecologismo y la antropología, siendo, de hecho, dos antropólogos sus grandes iniciadores y teóricos —Carlos Castaneda (1968, 1971, 1972 y 1974) y Michael Harner (1980)-.

Algunas de las disciplinas podrían englobarse en dos o más ámbitos ya que no siempre existe una frontera clara entre los distintos campos. Por ejemplo, el reiki, que he clasificado como terapia, podría situarse dentro del bloque de las espiritualidades orientales ya que sus practicantes tienen una alta orientación espiritual y practican meditación con regularidad. De la misma forma, el yoga o el taichi, por sus grandes beneficios sobre la salud psicofísica, podrían clasificarse perfectamente dentro del ámbito de las terapias.

\section{Prácticas y actividades}

Se han contabilizado un total de 180 locales, consultas y centros. En la mayoría de ellos se aplica, practica o enseña más de una disciplina, predominando las terapias naturales, que, de un modo u otro, están presentes en más de tres cuartas partes del total (150 locales). Las disciplinas orientales están presentes en un tercio de ellos (61), las prácticas esotéricas apenas constituyen una décima parte (21) y el neochamanismo solo se practica en 4 centros.

Las terapias alternativas son las que están presentes en más locales y centros, poseyendo además el abanico de técnicas más amplio. Así, un listado exhaustivo nos muestra la existencia de 80 tipos de técnicas o elementos relacionados con la medicina alternativa, lo que constituye más de la mitad del total de técnicas existentes en Tarragona, frente a una cuarta parte (34) en el campo de las espiritualidades orientales, a una décima parte (13) en el esoterismo y a unos pocos (2) en el neochamanismo.

El top ten de las actividades más expandidas lo constituyen los masajes (hasta 22 tipos diferentes, siendo el quiromasaje el más popular), el yoga (con hasta seis estilos diferentes), la reflexoterapia, la alimentación naturista, la homeo- 
patía, las flores de Bach, la fisioterapia, el reiki, la naturopatía y la acupuntura. El top ten de las terapias naturales es el mismo, pero con el drenaje linfático en último lugar. Por debajo hallamos terapias más desconocidas: terapia craneosacral, respiratoria, del sonido, de ultrasonidos, neural, energética, regresiva e integrativa, spa, cromoterapia, teatroterapia, aromaterapia, auriculoterapia, conciertoterapia, chakroterapia, vinoterapia, cerezoterapia, oligoterapia, arteterapia, acuaterapia, risoterapia, presoterapia, chocolaterapia, fangoterapia, magnetoterapia, laserterapia, electroterapia, termoterapia, drenaje linfático, quiropraxia, sales de Schüssler, rebirthing, cuencos de cuarzo, ventosas, macrobiótica, hipnosis, sanergía, osteopatía, kinesiología, iridología, sat nam rasayan, medicina china, medicina ayurvédica, digitopuntura tailandesa, sanación reconectiva, técnica Alexander y técnica metamórfica. De hecho, atendiendo solo al nombre, uno tiene la impresión de que cualquier cosa puede ser convertida en una terapia. También existen terapias que ejercitan el cuerpo, sin ser un deporte: microgimnasia, biodanza, aquagym, bioenergética, tonificación de los músculos, estiramientos asistidos, gimnasia correctiva, feldenkrais y chi kung. Perfectamente podríamos añadir aquí las artes marciales, el taichi o el yoga. Las fronteras son tenues. Por último, hallamos técnicas terapéuticas de corte psicológico: constelaciones familiares, coaching, PNL (programación neurolingüística), crecimiento personal, gestalt, psicoastrología y nueva consciencia, y las psicologías transpersonal, humanista, de eneatipos y del autoconocimiento.

El rango de las actividades englobadas dentro de las espiritualidades orientales es algo menor que el de las terapias. Lo más popular es el yoga, con varios estilos: hatha yoga de Sivananda y de Iyengar, kundalini yoga de Yogui Bhajan, acroyoga, pilates, yogalates y yoga tibetano. Es tan popular que está enfocado a todo tipo de público: adultos, niños, embarazadas, ancianos y hasta a empresas y colectivos. Las artes marciales constituyen otro de los campos populares y expandidos de la ciudad, con la presencia de varios gimnasios dedicados exclusivamente a ellas y con artes marciales japonesas, chinas, coreanas, indonesias y tailandesas. Tenemos así las tradicionales kárate, judo, taekwondo y kung fu, pero también otras menos conocidas como el ninjitsu, el kenjutsu, el tai-jitsu, el jodo, el aikido o el boxeo tailandés. Algunos estilos de taichi como el chen pueden incluirse en este apartado.

También hallamos la presencia de algunas danzas orientales como la danza del vientre, la de Bollywood o la shakti dance, si bien lo más habitual es hallar- 
las englobadas en el genérico "danza oriental". Muchas veces, por aquello del exotismo, se las encuentra junto a danzas africanas o de las llamadas "tribales". Entre las técnicas orientales no podía faltar la meditación: zen, tibetana, sobre los chakras o las concebidas por Osho (Bhagwan Shree Rajneesh), si bien la mayoría de veces simplemente se anuncia como "meditación", sin especificar. Otras técnicas o campos relacionados con lo oriental con presencia en la ciudad son el feng shui, la relajación, los mandalas, el neotantra y la venta de objetos y ropa oriental.

En el campo del esoterismo el abanico de disciplinas y técnicas es menor, siendo las mancias las más populares. Entre ellas, las que siguen teniendo más demanda son las dos más tradicionales: tarot y astrología. A cierta distancia en popularidad y aceptación hallamos la numerología, la quiromancia y las runas. Destaca igualmente la venta de objetos o libros relacionados con esta temática. De ellos, las velas para rituales serían lo más sobresaliente, aunque también las figuritas y los amuletos. Entre las técnicas de tipo esotérico a destacar encontraríamos los registros akáshicos (técnica canalizadora inspirada en la teoría teosófica que postula la existencia de campos de memoria colectiva llamados registros akáshicos) y los rituales de magia blanca. También podríamos incluir aquí otras técnicas como la psicoastrología o el feng shui. En cuanto a grupos de tipo esotérico, solo hemos hallado un par: la Sociedad de Estudios e Investigaciones Filosóficas (SEIF) y el Centro de Estudios de Autoconocimiento (CEA). Hay constancia también de un grupo masónico, la logia Mare Nostrum, pero en su página web ${ }^{1}$ no especifican la dirección.

Por último, el ámbito del neochamanismo es el que menor presencia tiene en la ciudad y casi todo es de tendencia americana, especialmente andina. Se ha constatado también la presencia de chamanismo africano en forma de magos que ofrecen servicios de rituales para propiciar salud, trabajo o amor y limpiezas energéticas de domicilios. $\mathrm{Al}$ anunciarse únicamente en pequeños carteles sin dirección donde solo consta el teléfono, no se les ha podido tener en cuenta a la hora de situarlos en el plano, por lo que no aparecen reflejados en los demás datos del artículo.

1. <http://www.mn25.org/>. 


\section{Los tipos de local}

En un primer análisis de las actividades de acuerdo con el lugar donde se llevan a cabo, se observa que la mayoría se realizan en locales especializados. Por su número, los centros y las consultas privadas constituyen la norma. Lo más habitual es que la actividad se produzca en un lugar que solo ofrece ese tipo de actividad y que, si bien es claramente un negocio, no se presente ni perciba como tal. Así, de acuerdo con el ámbito temático, en el campo de las terapias alternativas hallamos consultas médicas privadas donde el profesional está especializado en un tipo de terapia concreto, pero también centros donde se ofrece una gama más amplia de terapias y donde es habitual que atiendan y trabajen varios profesionales. Algunos centros incluso ofrecen cursos de formación en esas disciplinas y terapias. En cuanto a las espiritualidades orientales, se imparten especialmente en gimnasios y centros de yoga, o bien en domicilios privados cuando se trata de la meditación. En el ámbito del esoterismo, tenemos sobre todo consultas de adivinación (tarot y astrología) y tiendas dedicadas a la venta de objetos relacionados. Por último, el neochamanismo se halla siempre en centros multidisciplinares. Este último tipo de local combina disciplinas de dos o más ámbitos, habitualmente terapias y espiritualidad oriental. El mejor ejemplo de la ciudad es el Centro Holos.

Un segundo tipo de locales son aquellos que, poseyendo una orientación puramente económica, mantienen un enfoque exclusivo hacia los nuevos imaginarios culturales y que a efectos prácticos podemos denominar comercios exclusivos: tiendas, restaurantes vegetarianos y librerías. Son tiendas de alimentación y productos de higiene de producción biológica y tiendas de objetos de temática relacionada con los nuevos imaginarios culturales. Estas últimas se orientan a la venta de objetos de los ámbitos esotérico y terapéutico (cristales, cuencos tibetanos, cartas, etc.) o de la espiritualidad oriental (ropa, incienso, estatuas, etc.). Aunque los restaurantes vegetarianos no están enfocados exclusivamente a un cliente específico, mucha de su clientela habitual se mueve en el ámbito de la ecología, las terapias naturales o las espiritualidades orientales. En cuanto a las librerías, solo tenemos un ejemplo en la ciudad, y es de temática esotérica.

El último tipo de local lo constituirían los comercios que ofrecen servicios relacionados con los nuevos imaginarios culturales y que a efectos prácticos de- 
nominaremos comercios con servicios. Ejemplos típicos de este tipo de negocios son las peluquerías y los centros de estética que ofrecen a sus usuarios masajes y reiki, las academias de baile y los gimnasios que ofrecen danza del vientre o clases de yoga, o las farmacias que ofrecen remedios homeopáticos o sales de Schüssler.

\subsection{Análisis por el tipo de local}

De los 180 locales dedicados a estas temáticas y distribuidos por toda la ciudad, más de la mitad (101) son centros, algo más de un tercio (51) son comercios con servicios de los nuevos imaginarios culturales y algo más de una décima parte (28) son comercios exclusivos.

En un examen más detenido observamos que, de los 101 centros, más de la mitad (54) están dedicados exclusivamente a las terapias naturales, en torno a una cuarta parte (28) son centros multidisciplinares, algo más de una décima parte (15) están dedicados exclusivamente a la espiritualidad oriental y solo 4 se dedican únicamente al mundo de lo esotérico. De los 28 centros multidisciplinares, 20 combinan terapias con espiritualidad oriental; 4, terapias con espiritualidad oriental y esoterismo; 2 , terapias con esoterismo y neochamanismo; y otros 2, terapias, esoterismo, neochamanismo y espiritualidad oriental. De los 51 comercios con servicios, 15 (más de una cuarta parte) están dedicados a la estética, 13 son farmacias, 10 son peluquerías, 7 son escuelas de baile y 6 son gimnasios de fitness. De los 28 comercios exclusivos, más de la mitad (15) están orientados a las terapias. Las otras dos cuartas partes están dedicadas al mundo esotérico (7) y a la espiritualidad oriental (6).

¿Qué nos dice todo esto? Pues que en los nuevos imaginarios culturales predomina el ámbito terapéutico. En efecto, de los 180 locales, más de una cuarta parte (54) están dedicados exclusivamente a las terapias. Si además añadimos los 28 centros multidisciplinares con presencia de actividades terapéuticas, los 15 comercios exclusivos y los 38 comercios con servicios (15 salones de belleza, 13 farmacias y 10 peluquerías), tenemos que las terapias alternativas están presentes en tres cuartas partes de los locales.

Pese a la importancia conceptual de las espiritualidades orientales en los nuevos imaginarios culturales, su presencia en locales destinados a ellas en la ciudad de Tarragona está muy por detrás del ámbito terapéutico. Así, si su- 
mamos los locales donde se hallan presentes -15 centros exclusivos, 6 centros multidisciplinares, 6 comercios exclusivos y 38 comercios con servicios (15 centros de belleza, 10 peluquerías, 7 salones de baile y 6 gimnasios de fitness)—, obtenemos un total de 65 locales, es decir, algo más de una cuarta parte del total, muy por detrás del ámbito terapéutico.

El esoterismo y el neochamanismo muestran unos índices de presencia bastante bajos. Así, el esoterismo solo tiene presencia en algo más de una décima parte de todos los locales (4 centros exclusivos, 12 centros multidisciplinares y 7 comercios exclusivos). Por su parte, el neochamanismo solo tiene presencia en unos pocos centros multidisciplinares (4).

Por otro lado, los datos nos indican también el predominio de las mujeres en el mundo de los nuevos imaginarios culturales. De los 51 comercios con servicios, la mayoría son actividades femeninas como salones de estética (15) o peluquerías (10). A eso hay que añadir las actividades que, si bien están abiertas a ambos sexos, son más practicadas por mujeres, como los bailes ofrecidos por 7 escuelas, lo que constituye casi la mitad del total (32). Significativamente, entre esos comercios no hay ninguno dirigido exclusivamente a un público masculino. Todo ello corrobora lo ya expuesto por el GRIC (2012: 339-43).

\subsection{Análisis por ámbito temático}

$\mathrm{Al}$ analizar las disciplinas y actividades desde el punto de vista del campo temático, observamos que el ámbito de las terapias es el que cuenta con más tipos de locales y en mayor número. Se hallan presentes tanto en centros (exclusivos y multidisciplinares) y consultas privadas como en comercios exclusivos: tiendas de dietética y productos biológicos y tiendas de objetos esotéricos donde se venden objetos o utensilios usados en algunas terapias alternativas. Incluso han penetrado en locales muy diferentes de su tipo tradicional como centros de estética, peluquerías, academias de baile o gimnasios.

Las espiritualidades orientales se hallan presentes sobre todo en centros exclusivos para su aprendizaje y práctica (centros de yoga y meditación y gimnasios de artes marciales), pero también en centros multidisciplinares. En el ámbito comercial las hallamos en tiendas de objetos orientales y de alimentación vegetariana, pues la mayoría de ellas aconsejan el vegetarianismo como opción dietética. También han penetrado, aunque en menor grado que las terapias, en 
comercios que las ofrecen como servicio extra: centros de belleza (yoga, taichi, meditación o reiki), academias de baile (yoga, pilates o danzas orientales) y gimnasios (yoga o pilates).

El esoterismo tiene pocos locales exclusivos para su estudio y práctica — tan solo dos centros gnósticos y una logia masónica, algunas consultas privadas de tarot y un centro de parapsicología-, si bien también está presente en algunos centros multidisciplinares. En su vertiente comercial tenemos una librería y algunas tiendas de objetos mágico-esotéricos o de velas. Sin embargo, a diferencia de las terapias y las espiritualidades orientales, no lo hallamos en comercios con servicios. La excepción a la regla la constituye una herboristería, donde se ofrecen servicios de tarot y astrología.

Por último, el neochamanismo es el que tiene menor presencia. Tan solo se halla en centros multidisciplinares, donde es ofrecido como una disciplina más del amplio abanico a disposición del usuario. Incluso se halla ausente del ámbito comercial, a excepción de libros o merchandising en tiendas de objetos esotéricos. Quizá ello se deba a que, en la medida de lo posible, para su práctica se busca el contacto con la naturaleza, por lo que con frecuencia se trata de una actividad de fin de semana o de períodos vacacionales ofrecida en alguna de las casas rurales de la provincia dedicadas al mundo de lo alternativo.

\section{5. Áreas de distribución}

Al observar la distribución de los locales relacionados de una forma u otra con el mundo de los nuevos imaginarios culturales, lo primero que llama la atención es la existencia de una zona de máxima concentración en la parte más céntrica de la ciudad. Es una amplia área que, a grosso modo, abarcaría varias manzanas a ambos lados de la Rambla Nova, entre la plaza Imperial Tàrraco y el Balcón del Mediterráneo. ${ }^{2}$ Aquí los locales se hallan cerca de áreas comerciales (Rambla Nova, Mercado Central, área peatonal de Comte de Rius) o situados en arterias importantes o cercanas a ellas (Pere Martell, Prat de la Riba-Gasòmetre, Rovira i Virgili o avda. Catalunya).

Junto a la zona de máxima concentración central se dan también pequeñas áreas de concentración de locales. En el marco de Tarragona son seis: área de la Part Alta, en la zona derecha del barrio; área de la estación de ferrocarriles, cuyos

2. Véase el mapa. 
locales se concentran en las calles Orosi, Pau del Protectorat y Apodaca; área de la Marina, entre Pere Martell y plaza dels Infants; área de la avenida Andorra, comprendida entre la avenida Andorra y la calle Sant Antoni Maria Claret; área de Lluís Companys, cuyos locales se concentran en la avenida homónima; y área del Hospital Joan XXIII, entre ese hospital y la avenida Francesc Macià. El hecho de que las pequeñas áreas de concentración estén alejadas de la zona de máxima concentración central parece responder a la búsqueda de un público residente en dichas zonas. Si miramos con más detalle cada pequeña área de concentración, veremos que el patrón interno es similar o igual al de la zona de máxima concentración. Es decir, los locales se distribuyen dentro del barrio siguiendo las principales arterias de este o junto a sus áreas más comerciales. Así, en el barrio de la Marina sigue la arteria Pere Martell-Reial; en la zona de la estación de ferrocarriles, el eje Orosi-Apodaca; en la Part Alta, las calles Major, Merceria y Santa Anna, las más importantes del barrio y muy próximas a las tres plazas más comerciales de la Part Alta (plaza de la Font, del Rei y del Fòrum); en la zona de Joan XXIII, la avenida Francesc Macià y la plaza de les Corts Catalanes; y en la zona de las avenidas Andorra y Lluís Companys, las vías homónimas. Esta última junto al Corte Inglés y el Mercadona.

El resto de comercios son locales aislados. Así, fuera de la zona de máxima concentración y de las pequeñas áreas de concentración, encontramos 12 locales aislados en los barrios de la Marina (calles Torres Jordi, Smith y Reial), el Serrallo (calle Gravina), Part Alta (calles Descalços y Camí de la Cuixa) y Vall de l'Arrabassada (calles Joan Fuster y Josep Pla), y en las calles Joan Baptista Plana, Sant Auguri, avenida Roma y Via Augusta. Son la excepción que confirma la regla. Su distribución probablemente se deba a la asequibilidad de los alquileres — al estar fuera del área central—y a la búsqueda de público residente en esas áreas, como sucede con las pequeñas áreas de concentración. De hecho, los locales aislados se hallan siempre cerca de dichas áreas o en zonas intermedias entre ellas y la zona de máxima concentración. Por ejemplo, los locales aislados de las calles Descalços y Camí de la Cuixa se hallan próximos a la pequeña área de concentración de la Part Alta; los de las calles Smith y Reial, a la pequeña área de concentración del barrio de la Marina; y el de Joan Baptista Plana, al de la avenida Lluís Companys. 


\subsection{Distribución de los tipos de locales en las diferentes áreas}

Según su distribución, tres cuartas partes de los locales (138 de los 180 catalogados) se hallan en la zona de máxima concentración, 30 en las seis pequeñas áreas de concentración mencionadas y 12 son locales aislados. Entre los 138 locales de la zona de máxima concentración, más de la mitad (81) son centros, una cuarta parte (37) son comercios con servicios y algo más de una décima parte (20) son comercios exclusivos. En las pequeñas áreas de concentración la dinámica se invierte, predominando los comercios con servicios (12), seguidos de centros y consultas (10) y de comercios exclusivos (8). En cuanto a los 12 locales aislados, siguen una dinámica similar a la zona de máxima concentración. La mayoría (10) son centros o consultas; de ellos, 4 están dedicados exclusivamente a las terapias, 3 a las disciplinas orientales y otros 3 a una combinación de terapias y espiritualidades orientales. Los 2 locales restantes son farmacias, es decir, comercios con servicios.

\section{Dinámica de crecimiento}

Con los resultados del análisis anterior podemos intentar elucidar una posible dinámica de crecimiento y evolución en la ciudad en lo que a locales relacionados con los nuevos imaginarios culturales se refiere. Creo que, sin temor a equivocarnos, lo primero que se produce es la apertura de locales individuales, sean centros o consultas. Poco a poco en torno al área de alguno de esos centros se van abriendo otros, hasta producirse una pequeña área de concentración. Cuando se juntan varias de estas áreas, se produce una zona de máxima concentración. En el caso de Tarragona se da en la parte central de la ciudad, cuyo eje principal es la Rambla Nova. Es posible que en otras ciudades suceda lo mismo y que la primera zona de máxima concentración se dé también en el área central de la ciudad. Si así fuese — cosa bastante probable-, podríamos decir que sería una característica de su distribución. Tendríamos entonces una dinámica evolutiva en tres fases: 
Primera fase: apertura de locales aislados

Según los datos anteriores, en el caso de la ciudad de Tarragona lo primero que se abren son centros, principalmente consultas o centros dedicados exclusivamente a las terapias o a las espiritualidades orientales, y en algunos casos centros multidisciplinares donde se combinan los dos ámbitos. Algunos comercios normales empiezan a ofrecer productos o servicios relacionados con los nuevos imaginarios culturales. En nuestro caso son farmacias que ofrecen productos de medicina alternativa, que suponen solo algo más de una décima parte del total de la actividad. Estos primeros locales suelen estar en o cercanos a alguna de las principales arterias, o cerca de áreas comerciales de la ciudad o de la zona de apertura.

En esta primera fase hacen su aparición los ámbitos de las terapias y la espiritualidad oriental, así como los centros (exclusivos y multidisciplinares) y los comercios con servicios. Estos ámbitos y tipos de local son ya desde el principio el motor de los nuevos imaginarios culturales.

\section{Segunda fase: pequeñas áreas de concentración}

En torno a algunos de esos centros se empiezan a abrir nuevos locales, hasta producir una pequeña área de concentración. En estas áreas los tipos de locales que predominan no son los centros o consultas, sino los comercios con servicios. En el caso de Tarragona priman por igual farmacias y salones de estética, seguidos por escuelas de baile y peluquerías.

Los centros y las consultas ocupan el segundo lugar en número de locales. Siguen predominando los centros dedicados exclusivamente a las terapias (90), seguidos ahora por los centros multidisciplinares (54) — cuya fórmula combinatoria más frecuente es combinar terapias con espiritualidad oriental (36) o con espiritualidad oriental y esoterismo (18) - y por los centros dedicados exclusivamente a la espiritualidad oriental (36).

En las pequeñas áreas de concentración aparecen primero los comercios exclusivos. Probablemente ello se deba a la necesidad previa de un público lo suficientemente numeroso como para poder sostenerlo. En el caso de Tarragona predominan los comercios enfocados a las terapias naturales, sobre todo tiendas de alimentación naturista. Luego siguen aquellos dedicados a la espiri- 
tualidad oriental y al esoterismo, mayormente tiendas de objetos dedicados a uno u otro ámbito (estatuas, incienso, velas, etc.).

En esta segunda fase aparecen por primera vez el ámbito del esoterismo (ligado a los centros multidisciplinares) y los comercios exclusivos.

\section{Tercera fase: zona de máxima concentración}

En esta fase varias de las pequeñas áreas de concentración crecen hasta juntarse, produciendo una zona de máxima concentración. En el caso de Tarragona esta zona se encuentra en el centro de la ciudad, cuyo eje central sería la Rambla Nova. Al igual que en la primera fase, predominan los centros, seguidos de los comercios con servicios, si bien los comercios exclusivos siguen en tercer lugar tal y como sucedía en la segunda fase.

La evolución del tipo de centro sigue la misma dinámica que en las otras dos fases, predominando así centros o consultas dedicados a las terapias alternativas, seguidos de centros multidisciplinares y de centros de espiritualidades orientales. Aquí la novedad estriba en la aparición de centros dedicados exclusivamente a actividades del ámbito del esoterismo. En cuanto a la combinación de ámbitos en los centros multidisciplinares, se mantienen las dos primeras combinaciones (terapias + espiritualidad oriental / terapias + espiritualidad oriental + esoterismo) y se producen otras dos nuevas (terapias + espiritualidad oriental + esoterismo + neochamanismo / terapias + esoterismo + neochamanismo). Es decir, aparece un nuevo ámbito: el neochamanismo. Este, como vemos, va ligado a centros multidisciplinares, combinado siempre con las terapias $y$ el esoterismo, curiosamente sus dos ámbitos más cercanos. No parece casual.

Los negocios con servicios vuelven al segundo lugar, pero ya no predominan las farmacias (total de locales en la primera fase y un tercio en la segunda), sino los salones de estética (casi un tercio del total en esta fase). Le siguen las peluquerías (la cuarta parte) y las farmacias (menos de la cuarta parte). La novedad reside en la aparición de un nuevo tipo de comercio: los gimnasios de fitness. En último lugar tenemos las academias de baile, que suponen una décima parte del total. Así, en la evolución de los comercios con servicios solo aparecen farmacias en la primera fase. Luego aparecen con fuerza los salones de belleza - que igualan a las farmacias - y las escuelas de baile, además de las peluquerías. Por último, los centros de belleza y estética pasan a la cabeza, seguidos de las pelu- 
querías y de las farmacias, que siguen bajando puestos y porcentajes. También irrumpen con cierta fuerza los gimnasios de fitness, que se sitúan por delante de las escuelas de baile.

Los comercios exclusivos, que aparecieron en la segunda fase, en la que la mitad de ellos estaban dedicados a las terapias, una cuarta parte a las espiritualidades orientales y la otra cuarta parte al esoterismo, se mantienen prácticamente igual. Así, los comercios orientados a las terapias naturales aumentan ligeramente por encima de la mitad, el esoterismo mantiene su segunda posición con un cuarto del total y los comercios enfocados a las espiritualidades orientales disminuyen ligeramente por debajo de la cuarta parte.

\section{Dinámica de crecimiento: el caso de las espiritua- lidades orientales}

Examinaremos ahora la dinámica de implantación y crecimiento analizada en el apartado anterior a partir del caso de las espiritualidades orientales para comprobar hasta qué punto se cumple o no el modelo propuesto.

Podemos establecer con claridad tres etapas en el desarrollo de los locales relacionados con los nuevos imaginarios culturales. La primera, en las décadas de los setenta y ochenta, se caracteriza por la apertura de los primeros gimnasios de artes marciales y centros de yoga de la ciudad. En la segunda, en los noventa, se abrieron los primeros centros de meditación y llegaron nuevos estilos de yoga y artes marciales. La última, ya en este siglo xxi, viene marcada por la aparición de nuevas formas de meditación y estilos de yoga, con una expansión que sobrepasa los locales tradicionales, penetrando con fuerza en centros de terapias alternativas y multidisciplinares y en negocios con servicios (escuelas de danza, centros de belleza y gimnasios de fitness).

\subsection{La etapa de asentamiento: apertura de locales aislados}

En las décadas de los setenta y ochenta, debido a la serie Kung Fu y a la influencia del cine de Hong Kong, se abrieron los primeros gimnasios de artes marciales. La introducción de las artes marciales se produjo de la mano de orientales que, hallándose aquí y percibiendo la demanda existente creada por el mundo del cine y la televisión, decidieron fundar sus respectivos gimnasios. Un 
japonés llamado Hoyo abrió el gimnasio de judo Yodokara (1975; c/ Sant Antoni Maria Claret); Kozo Mizoguchi, los gimnasios de kárate Musoken (1978; c/ Jaume I) y Kushinkai (principios de los ochenta; c/ Doctor Zamenhof); y el maestro coreano Chois Cha empezó a enseñar taekwondo en el desaparecido polideportivo Elida (avda. Andorra) en 1975, fundando su propio gimnasio en 1980 (c/ Joana Jugan). El Club de Judo Yodokara cerró en 1983, y su profesor de entonces, Josep Esteve, fundó ese mismo año el Club de Judo Tarragona en Taquígraf Martí (Planells, 2009: 204-5).

El establecimiento del yoga en Tarragona, que tuvo lugar durante esa misma época, se debió a nativos que se formaron como profesores en el extranjero y decidieron dedicarse al yoga de forma profesional. Sus introductores fueron Puri, Jaume Guasch y Xavier Montserrat, todos ellos formados en Canadá. Puri inició su andadura en 1979 en una escuela de danza ya desaparecida (Ballet Estudio; c/ Méndez Núñez). Durante un tiempo tuvo allí su escuela y hoy se dedica a impartir clases en diferentes lugares. Ese mismo año Jaume Guasch fundó el Yoga International Institute en Pin i Soler, germen de lo que más tarde se transformaría en el centro multidisciplinar Holos. Xavier Montserrat empezó dando clases en gimnasios de artes marciales como Musoken y en 1984 abrió su propio centro (Yogananda, en la calle Unió). Durante esa etapa llegaron también el chi kung y el taichi, cuyas primeras clases se impartieron en centros de yoga.

\subsection{La primera expansión: formación de pequeñas áreas de concentración}

En la segunda etapa, que tuvo lugar en la década de los noventa, se produjeron las primeras pequeñas áreas de concentración, caracterizadas por la llegada de métodos de meditación y la ampliación de la oferta de técnicas. Así, a principios de los noventa se abrieron nuevos gimnasios de artes marciales como Kuntao (c/ Mallorca), que ofrecía kung fu indonesio y taichi, o Budo Shin (1992; c/ del Mar), que ofrece tai-jitsu, ninjitsu, iaido, jodo y aikido. También llegaron versiones más marciales del taichi (estilo chen) a gimnasios de kárate como Kushinkai. Por otro lado, el taichi se expandió a centros de terapias y a centros multidisciplinares como Holos. 
Se abrió un centro multidisciplinar, Els Àngels (plaza dels Àngels), en el casco antiguo de Tarragona, donde se impartían clases de hatha yoga y terapias. En 1995 se introdujo un nuevo estilo, el kundalini yoga de Yogui Bhajan, que empezó impartiéndose en el centro de yoga de Puri (c/ Méndez Núñez). De allí se trasladó a la Part Alta (c/ de la Nau) y luego cerca del Mercado (c/ Lleida), donde continúa con el nombre de Atma. Su introductor fue Prasad, formado en Barcelona. Continuó con la actividad una alumna suya, Joaquina, que también se formó en Barcelona.

La meditación zen aterrizó en 1992 de la mano de Ramón Costa, por entonces estudiante de acupuntura formado como estudiante de meditación zen en Francia (zen de la escuela soto del maestro Taisen Deshimaru), que abrió un dojo primero en la c/ Talavera y luego en la c/ Calderers, ambas cerca de la plaza del Fòrum.

A mediados de los noventa llegó también una nueva forma de espiritualidad oriental llamada energía universal, que tuvo su centro en la Part Alta (baixada de la Peixateria). Se trataba de una forma de sanación por imposición de manos procedente de Corea del Sur e introducida por un vietnamita llamado Curtis Cao, algo similar al reiki. Después de una fallida profecía en torno al año 2000, el grupo, acusado de sectario, fue intervenido por la policía y acabó desapareciendo, y con este el centro (Fuentes, 2000).

En cuanto a los comercios de temática oriental, entre los ochenta y los noventa se abrieron dos restaurantes vegetarianos, de los cuales solo subsiste $\mathrm{Al}$ Natural (c/ Arquitecte Rovira); el otro estaba en la plaza de Fòrum. Los dos restaurantes chinos de entonces ( $\mathrm{Pa}$-li y Xanghai) se engloban también dentro de este ámbito. Ambos estaban en la misma zona, uno en la calle Girona y el otro en la Rambla Nova. En la plaza del Rei había una tienda de piedras y objetos esotéricos (Yeha Noha), junto a una librería de espiritualidad (Arcoal), y en Prat de la Riba, una tienda de objetos orientales, ya desaparecida.

De este modo se constituyeron las primeras pequeñas áreas de concentración. Una en la zona del Baró de les Quatre Torres (Judo Tarragona, Holos); otra cerca de la plaza de toros (tienda de Prat de la Riba y gimnasios Musoken, Kushinkai y Kuntao); otra entre la calle Unió y Méndez Núñez (centros de yoga de Puri y Yogananda); una tercera al principio de la Rambla Nova (restaurantes chinos Xanghai y Pa-li); y una cuarta en la Part Alta (dojo zen, Els Àngels, Arcoal, Yeha Noha y restaurante vegetariano de la plaza Fòrum). 


\subsection{La agrupación de las pequeñas áreas de concentración en una zona de máxima concentración central}

En esta última etapa han llegado nuevos estilos de yoga: hatha yoga del estilo Iyengar (dos centros), pilates, yogalates, acroyoga y hatha yoga tibetano. Se han abierto dos nuevos locales de kundalini yoga (avda. Roma y c/ Ixart), y este yoga se ha introducido en algunos centros multidisciplinares (Els Àngels) y sociales (Kal Pobre) de la Part Alta, así como en algunos centros de terapias.

Han aparecido grupos de meditación tibetana y vipassana. La primera forma de meditación tuvo presencia en una asociación de mujeres situada al principio de la Rambla Nova, pero por problemas económicos se trasladó a domicilios particulares. La meditación vipassana se realizaba en un domicilio hasta que el grupo se disolvió por desavenencias. En la actualidad hay constancia de la existencia de un nuevo grupo, pero a nivel de la provincia, fuera de la ciudad. Han aparecido asimismo nuevas técnicas de meditación como la meditación en los chakras o las meditaciones de Osho (Bhagwan Shree Rajneesh). La meditación, sin especificar el tipo, se introduce en varios centros de terapias y consultas. El reiki, disciplina terapéutico-espiritual japonesa, se introduce en numerosos centros de terapias alternativas y en centros de belleza y peluquerías. Incluso se crea una asociación, Cobquecura (avda. Ramón y Cajal).

En esta etapa las actividades ligadas al ámbito de lo oriental se expanden por la ciudad, sobre todo a centros multidisciplinares y de terapias alternativas, pero también a escuelas de baile, centros de belleza y peluquerías. Junto a las técnicas anteriores aparecen las danzas (del vientre, de Bollywood y oriental) y el feng shui, pero también medicinas o técnicas terapéuticas: medicina china, ayurvédica, digitopuntura, masaje tailandés, auriculoterapia, macrobiótica, sat nam rasayan, terapia de cuencos tibetanos y shiatsu. En el campo de los comercios exclusivos, se abren cuatro tiendas de objetos orientales (tres de ellas en Comte de Rius y una en la Part Alta). Este boom es atribuible a la época de bonanza que acompañó el inicio de siglo (la crisis ha acabado con varios comercios) y conllevó que las pequeñas áreas de concentración, antes aisladas, produjesen una zona de máxima concentración en el centro de la ciudad. Así, a grandes rasgos, la implantación y dinámica de crecimiento de la espiritualidad oriental en Tarragona confirma el patrón establecido anteriormente. 


\section{Conclusiones}

De los análisis anteriores podemos constatar la existencia de tres tipos básicos de local: centros y consultas, que pueden ser exclusivos cuando solo actúan en un ámbito o multidisciplinarios cuando combinan técnicas de dos o más ámbitos; comercios exclusivos; y comercios con servicios. Estos locales se distribuyen por la ciudad de tres formas diferentes: formando una zona de máxima concentración en el centro de la ciudad, en pequeñas áreas de concentración relativamente alejadas del centro o como locales aislados. Su crecimiento se produciría en tres fases:

1. Apertura de locales aislados:

- Aparecen los ámbitos de las terapias y las espiritualidades orientales.

- Se abren consultas y centros exclusivos de un ámbito o multidisciplinares.

- Se abren comercios con servicios.

2. Surgimiento de pequeñas áreas de concentración:

- Aparece el ámbito del esoterismo.

- Se abren comercios exclusivos.

3. Producción de una zona de máxima concentración:

- Aparece el ámbito del neochamanismo.

- No aparece ningún tipo de local nuevo, pero sí subtipos nuevos:

- Centros dedicados exclusivamente al ámbito esotérico.

- Gimnasios de fitness dentro de los comercios con servicios.

De lo anterior se infiere que el motor de los nuevos imaginarios culturales son los centros exclusivos y multidisciplinares dedicados a las terapias y/o las espiritualidades orientales. Luego, en este tipo de local se darán nuevos ámbitos como el esoterismo o el neochamanismo respondiendo a la ampliación del mercado original. En cuanto a los locales de tipo comercial, el motor inicial son los comercios con servicios. Tan solo cuando los locales aislados se afianzan y se transforman en pequeñas áreas de concentración se da la demanda suficiente que permite la existencia de comercios exclusivos.

La apertura de un local parece obedecer a dos variables: economía y centralidad. Es decir, búsqueda de un alquiler razonable y búsqueda de centralidad. Estas dinámicas se producen incluso cuando salimos de la zona de máxima concentración. Así, en las pequeñas áreas de concentración se busca situarse 
en o cerca de alguna arteria importante de la zona, es decir, en su parte más "central". Ello se debe al deseo de acercar estas técnicas a su principal cliente: la clase media. Esto explica también las zonas de las que se halla totalmente ausente o en las que tiene poca presencia, al ser percibidas como deprimidas o inseguras por esa clase. Sería interesante poder comparar en futuros estudios si esta dinámica sigue el mismo patrón de otros tipos de negocios dirigidos a esa misma clase media (informática, ocio cultural, viajes, etc.) o si se diferencia.

Recordemos también que las terapias alternativas están presentes en tres cuartas partes de los locales dedicados a los nuevos imaginarios culturales en Tarragona y las técnicas orientales en algo más de una cuarta parte. Teniendo en cuenta que casi la totalidad de las terapias tienen una base corporal y que las técnicas orientales (a excepción de la meditación) son también de base corporal (artes marciales, yoga, danza, etc.), podemos concluir que la espiritualidad de los nuevos imaginarios culturales posee una base mayoritariamente corporal. Esto nos indica las necesidades de una clase con trabajos más sedentarios debido a su mayor formación (GRIC, 2012:316-7).

Todo esto confirma que la espiritualidad de los nuevos imaginarios culturales es fundamentalmente una terapéutica espiritual de base corporal, lo cual viene a corroborar las conclusiones del GRIC (2012). Ahora bien, esto matiza conclusiones como las de F. Champion $(1989,1993$ y 1994) en cuanto a que la supuesta liquidez o nebulosidad del fenómeno quizá no lo sea tanto como se suponía en un principio. También corregiría la percepción del fenómeno como "místicoesotérico" pues, aun considerando su grado de indefinición y parafraseando a Champion, nos hallaríamos ante una espiritualidad "místico-terapéutica".

Por otro lado, la presencia marginal del ámbito del esoterismo en los nuevos imaginarios culturales en la ciudad de Tarragona no parece apoyar la sólida tesis de W. Hanegraaff (1998), que plantea que el núcleo de estos está en el ámbito esotérico. Ahora bien, la aparente contradicción de un fenómeno surgido del esoterismo pero que posee un indudable núcleo de espiritualidad terapéutica de base corporal y una presencia marginal de practicantes y técnicas esotéricas podría explicarse si, como intuyo, la mayoría de terapias alternativas tuviesen su base en los planteamientos y/o movimientos esotéricos que plantea Hanegraaff, si bien esta confirmación o refutación deberá quedar para más adelante. 


\section{Bibliografía}

Arntz, W., Chasse, B. y Vicente, M. (2006): ¿iY tú qué sabes!? Madrid: Palmyra.

Blavatsky, H. P. (1977 [1877]): Isis sin velo (4 vol.). Madrid: Eyras.

- (1978 [1888]): La doctrina secreta (6 vol.). Madrid: Luis Cárcamo.

Bailey, A. A. (1985): El discipulado en la nueva era. Buenos Aires: Kier.

Barker, E. (1994): “The New Age in Britain”, en J.-B. Martin y F. LaplanTINe (dirs.): Le défi magique, vol. 1. Lyon: Presses Universitaires de Lyon.

Вонм, D. (2008): La totalidad del orden implicado. Barcelona: Kairós.

Capra, F. (2005 [1975]): El Tao de la física. Málaga: Sirio.

Carozzi, M.J. (1999): "La autonomía como religión: la nueva era", en Alteridades (Antropología de los Movimientos Religiosos), 18, pp. 19-38.

Castaneda, C. (1995 [1968]): Las enseñanzas de Don Juan. México D.F.: Fondo de Cultura Económica.

- (1987 [1971]): Una realidad aparte. México D.F.: Fondo de Cultura Económica.

- (1985a [1972]): Viaje a Ixtlán. México D.F.: Fondo de Cultura Económica.

- (1985b [1974]): Relatos de poder. México D.F.: Fondo de Cultura Económica.

Champion, F. (1989): "Les sociologies de la post-modernité religiose et la nébuleuse mystique ésoterique", Archives des Sciences Socials des Religions, vol. 67, 1, pp. 155-169.

- (1993): "Religeux flottant, éclecticisme et syncrétismes", en J. Delumeau (ed.): Le fait religieux, pp. 741-777. París: Fayard.

- (1994): "La nébuleuse mystique-ésoterique: Une décomposition du religieux entre humanisme revisité, magique, psychologie”, en J.-B. Martin y F. LAPLANTINe (dirs.): Le défi magique, vol. 1,"Esoterisme, occultisme, spiritisme".

Fedele, A. (2008): El camino de María Magdalena. Un recorrido antropológico por la ruta de peregrinaje de la nueva espiritualidad. Barcelona: Integral/ RBA.

Ferguson, M. (1994 [1980]): La conspiración de acuario. Madrid: América Ibérica.

Fericgla, J. M. (1994): Plantas, chamanismo y estados de consciencia. Barcelona: La liebre de marzo. 
- (1997): Al trasluz de la ayabuasca. Antropología cognitiva, oniromancia y consciencias alternativas. Barcelona: La liebre de marzo.

Fort, C. (1969 [1919]): El libro de los condenados. Barcelona: Rumeu.

Fuentes, F. (2000): "Redada en Cataluña contra una secta coreana que prometía curar el cáncer”, El País, 23 de febrero.

GRIC / J. Prat (coord.) (2012): Els nous imaginaris culturals. Tarragona: Publicacions de la URV.

Grof, S. (1994): La mente holotrópica. Barcelona: Kairós.

- (2001): Psicología transpersonal. Nacimiento, muerte y trascendencia en psicoterapia. Barcelona: Kairós.

Hanegraaff, W.J.A. (1998): New Age Religion and western Culture: Esotericism in the Mirror of Secular Thought. Albany, Nueva York: State University of New York Press.

Harner, M. (1987 [1980]): La senda del chamán. Madrid: Swan.

Heelas, P. (1996): The New Age Movement: The Celebration of the Self and the Sacralization of Modernity. Oxford: Blackwell.

Huxley, A. (2004 [1945]): La filosofía perenne. Barcelona: Edhasa.

- (2006 [1954]): Las puertas de la percepción. Barcelona: Edhasa.

Kaku, M. (2010)ः Física de lo imposible. Barcelona: DeBolsillo.

Lenoir, F. (2003): Les Métamorphoses de Dieu. París: Plon.

Lewis, J.R. y Melton, J.G. (1992): Perspectives on the New Age. Albany, Nueva York: State University of New York Press.

Lovelock, J.E. (2007): Las edades de Gaia. Una biografía de nuestro planeta vivo. Barcelona: Tusquets.

- (2008): La venganza de la tierra. La teoría de Gaia y el futuro de la bumanidad. Barcelona: Planeta de Agostini.

McKenna, T. (1994): El manjar de los dioses. Una bistoria de las plantas, las drogas y la evolución bumana. Barcelona: Paidós.

- (2001): Alucinaciones reales. Relato de las extraordinarias aventuras del autor en el paraíso del diablo. Castellar de la Frontera, Cádiz: Castellarte.

Merlo, V. (2007): La llamada (de la) Nueva Era. Hacia una espiritualidad místico-esotérica. Barcelona: Kairós.

Narby, J. (1999): The Cosmic Serpent: DNA and the Origins of Knowledge. Nueva York: Jeremy P. Tarcher. 
Pauwels, L. y Bergier, J. (1994 [1960]): El retorno de los brujos. Madrid: América Ibérica.

Pinchieck, D. (2007 [2002]): Una historia de las drogas. Un viaje psicodélico al corazón del chamanismo contemporáneo. Barcelona: RBA.

- (2006): 2012: The Return of Quetzalcoatl. Jeremy P. Tarcher / Penguin. Nueva York.

Planells i Garcés, E. (2009): Fuentes documentales y testimoniales para el estudio de las artes marciales en España: Ju-Jutsu, Judo y Aikido. Barcelona: INEF.

Prat, J. y Moreno, J. (2007): “La nebulosa misticoesotèrica”, en Tradicionari, vol. 8, "L’Univers màgic. Mites i creences", pp. 278-289. Barcelona: Enciclopedia Catalana.

Pribram, K. y Martín, J. (1995): Cerebro y Conciencia. Madrid: Díaz de Santos.

Rätsch, C., Müller-Ebeling, C. y Shahi, S. B. (2002): Shamanism and Tantra in the Himalayas, Londres: Thame y Hudson.

Ravinovitch, S.T.y Reid, S.L. (2004): “Witches, wiccans, and Neo-Pagans: A Review of Current Academic Treatments of Neo-Paganism”, en J. R. Lewis (ed.): New religious Movements, pp. 514-532. Nueva York: Oxford University Press.

Schwartz, T. (2002): Lo que realmente importa. En busca de la sabiduría en Occidente. Barcelona: La liebre de marzo.

Sheldrake, R. (1989): Una nueva ciencia de la vida. La bipótesis de la causación formativa. Barcelona: Kairós.

Siqueira, D. (2004): "La laberíntica recerca religiosa en l'actualitat: creences i pràctiques místiques i esotèriques a la capital del Brasil”, en Revista d'Etnologia de Catalunya, 24, pp. 100-129.

Steiner, R. (1978): La ciencia oculta. Madrid: Ed. R. Steiner.

Тацвот, M. (2007 [1991]): El universo holográfico. Madridः Palmyra.

TART, C. (1979): Psicologías transpersonales. Buenos Aires: Paidós.

Washburn, M. (1999): Psicología transpersonal en una perspectiva psicoanalitica. Barcelona: La liebre de marzo.

Wilber, K. (1990): El espectro de la conciencia. Barcelona: Kairós.

- (1993): Psicología integral. Barcelona: Kairós. 